ON THE SOLUTION OF THE DIFFERENTIAL EQUATION, ETC.

Putting $x=0$ and dividing by $x^{\prime}$, we find that the point of intersection of the normals at $P$ and $Q$ is given by

$$
\left(y-y^{\prime}\right)\left(2 a+3 b x^{\prime}+\ldots\right)=1 \text {. }
$$

Putting $x^{\prime}$ and $y^{\prime}$ both zero, this gives in the limit

$$
y=\frac{1}{2 a} \text {. }
$$

Now, according to Newton's treatment, the radius of curvature is given by

$$
L t \frac{y}{x^{2}}=\frac{1}{2 \rho} \text { whence } \frac{1}{2 a}=\rho,
$$

and it follows that $y=\rho$ according to this definition.

The Newtonian method treats the circle of curvature at $P$ as the limiting position of a circle touching the curve at $P$ and passing through a point $Q$ which ultimately coincides with $P$.

This method is a little less general than the definition of the circle of curvature as the limiting position of a circle through three consecutive points. But it is a necessary condition for the existence of a circle of curvature that the circle through three points of a curve should tend to a unique limiting position, whatever be the law according to which the points approach coincidence, and this covers the case, contemplated in the Newtonian method, in which two of the points coincide before the third point coincides with them.

Personally, I have always regarded it as pure "fudge" to treat the definition quoted by $\mathrm{Dr} M$ 'Whan as equivalent to that based on considerations of the circle through three consecutive points of a curve. If such an assumption can be justified on any grounds, I should be interested to hear of them ; meanwhile I have my doubts.

$$
\text { G. H. Bryan. }
$$

\title{
On the Solutions of the Differential Equation
}

$$
\frac{d^{n} x}{d t^{n}}+P_{1} \frac{d^{n-1} x}{d t^{n-1}}+\ldots+P_{n-1} \frac{d x}{d t}+P_{n} x=0 .
$$

The following is a direct proof that any $n+1$ particular integrals of the differential equation

$$
x_{n}+P_{1} x_{n-1}+P_{2} x_{n-2}+\ldots+P_{n-1} x_{1}+P_{n} x=0,
$$


where

$$
x_{r} \equiv \frac{d^{r} x}{d t^{r}}
$$

and $P_{1}, P_{2} \ldots P_{n}$ are functions of $t$ alone, are connected by a linear relation.

Let us take $n=3$, and it will be seen that the same method will apply to any value of $n$, and let $x, y, z, u$ be four particular integrals.

We have

$$
\begin{aligned}
& x_{3}+P_{1} x_{2}+P_{2} x_{1}+P_{3} x=0 \\
& y_{3}+P_{1} y_{2}+P_{2} y_{1}+P_{3} y=0 \\
& z_{3}+P_{1} z_{2}+P_{2} z_{1}+P_{3} z=0 \\
& u_{3}+P_{1} u_{2}+P_{2} u_{3}+P_{2} u=0
\end{aligned}
$$

wherice

$$
\left|\begin{array}{llll}
x_{3} & x_{2} & x_{1} & x \\
y_{3} & y_{2} & y_{1} & y \\
z_{3} & z_{2} & z_{1} & z \\
u_{3} & u_{2} & u_{1} & u
\end{array}\right|=0
$$

For brevity write

Then

$$
\begin{aligned}
& \left|\begin{array}{ccc}
x & y & z \\
x_{1} & y_{1} & z_{1} \\
x_{2} & y_{2} & z_{2}
\end{array}\right|=U \text {, etc. } \\
& \frac{d U}{d t}=\mid \begin{array}{ccc}
x & y & z \\
x_{1} & y_{1} & z_{1} \\
x_{3} & y_{3} & z_{3}
\end{array}, \text { etc. }
\end{aligned}
$$

are well known; and the determinant $(A)$ at once gives

$$
x_{2} \frac{d X}{d t}+y_{2} \frac{d Y}{d t}+z_{2} \frac{d Z}{d t}+u_{2} \frac{d U}{d t}=0
$$

and clearly $\quad x_{1} \frac{d X}{d t}+y_{1} \frac{d Y}{d t}+z_{1} \frac{d Z}{d t}+\iota_{1} \frac{d U}{d t}=0$

$$
\begin{aligned}
& x \frac{d X}{d t}+\ldots \ldots \ldots \ldots \ldots \ldots \ldots \ldots \ldots=0 \\
& x_{3} \frac{d X}{d t}+\ldots \ldots \ldots \ldots \ldots \ldots \ldots \ldots \ldots=0 .
\end{aligned}
$$

But (A) also gives us

$$
\text { and, identically, } \begin{aligned}
& x_{2} X+y_{2} Y+z_{2} Z+u_{2} U=0, \\
& x_{1} X+y_{1} Y+\ldots \ldots \ldots \ldots=0, \\
& x X+y Y+\ldots \ldots \ldots \ldots=0 .
\end{aligned}
$$


Hence

$$
\begin{aligned}
& \frac{d X}{d t}=\frac{d Y}{d t}=\frac{d Z}{Y}=\frac{\frac{d U}{Z t}}{Z}=\frac{d t}{U} \\
\therefore \quad & \frac{X}{a}=\frac{Y}{b}=\frac{Z}{c}=\frac{U}{d}
\end{aligned}
$$

where $a, b, c, d$ are constants,
and since
$x X+y Y+z Z+u U=0$
we have
$a x+b y+c z+d u=0$,
which is the result required.

J. E. A. Steggall.

On the Solutions of $\frac{d^{2} u}{d t^{2}}+P \frac{d u}{d t}+Q u=0$.

(Suggested in discussion at a meeting of the Society).

The following note gives a proof of the theorem that if $x, y, z$ are any three values of $u$ which satisfy the differential equation $\frac{d^{2} u}{d t^{2}}+P \frac{d u}{d t}+Q=0$, then $l x+m y+n z=0$ where $l, m, n$ are constants. If the dots denote differentiation with respect to $t$, we have $\ddot{x}+P \dot{x}+Q x=0, \quad \ddot{y}+P \dot{y}+Q y=0$ and $\ddot{z}+P \dot{z}+Q z=0$.

Hence

$$
\left|\begin{array}{lll}
x & y & z \\
\dot{x} & y & z \\
\ddot{x} & \ddot{y} & \ddot{z}
\end{array}\right|=0 .
$$

Now $x, y, z$ are functions of $t$, and therefore the locus of the point $(x, y, z)$ is a curve in space. Equation (1) is the condition that the osculating plane at $(x, y, z)$ should pass through the origin. But if the osculating plane at any point of a curve passes through a fixed point, the curve is a plane curve lying in a plane through the point. Therefore the point $(x, y, z)$ lies in a plane through the origin or $l x+m y+n z=0$.

R. J. T. BeLL. 